\title{
Quasinormal Modes, the Area Spectrum, and Black Hole Entropy
}

\author{
Olaf Dreyer \\ Perimeter Institute for Theoretical Physics, 35 King Street North, Waterloo, Ontario N2J 2W9, Canada
}

(Dated: October 31, 2018)

\begin{abstract}
The results of canonical quantum gravity concerning geometric operators and black hole entropy are beset by an ambiguity labelled by the Immirzi parameter. We use a result from classical gravity concerning the quasinormal mode spectrum of a black hole to fix this parameter in a new way. As a result we arrive at the Bekenstein - Hawking expression of $A / 4 l_{P}^{2}$ for the entropy of a black hole and in addition see an indication that the appropriate gauge group of quantum gravity is $\mathrm{SO}(3)$ and not its covering group $\mathrm{SU}(2)$.
\end{abstract}

PACS numbers: 04.70.Dy, 04.60.Pp

\section{A. Introduction}

The canonical approach to quantum gravity (see [1] for a recent review) boasts two remarkable sets of results. It has quantum operators for area and volume that have discrete spectra and it puts forward a derivation of the entropy of a black hole. Both these results are plagued by the existence of one free parameter. Up to now the only way to fix this ambiguity was to use the result for the black hole entropy and adjust it to the Bekenstein Hawking result. This then also fixes the ambiguity in the spectra of the geometrical operators. In this note we will put forward an independent argument to fix the ambiguity rendering the black hole calculation a true prediction of the theory.

A basis for the Hilbert space of canonical gravity is given by spin networks. These are graphs whose edges are labelled by representations of the gauge group of the theory. In the case of gravity this group is taken to be $\mathrm{SU}(2)$ and the representations are thus labelled by positive half-integers $j=0,1 / 2,1,3 / 2, \ldots$ If a surface is intersected by an edge of such a spin network carrying the label $j$ the surface acquires the area [2, 3]

$$
A(j)=8 \pi l_{P}^{2} \gamma \sqrt{j(j+1)}
$$

where $l_{P}$ is the Planck length and $\gamma$ is the so called Immirzi parameter [4]. This is the ambiguity we spoke of before. It parameterizes an ambiguity in the choice of canonically conjugate variables that are to be quantized. There is no a priori reason to fix this parameter to any particular value.

The only argument so far that could be used to fix this parameter comes from black hole entropy. Given a black hole horizon one can think of the area of the horizon as being a consequence of a large number of spin network edges puncturing the surface. (For a detailed account of black hole entropy in canonical quantum gravity see [5, 6, 7, 8, 9]. The review [1] and the citations contained therein are also helpful.) Each edge with spin $j$

*Electronic address: odreyer@perimeterinstitute.ca

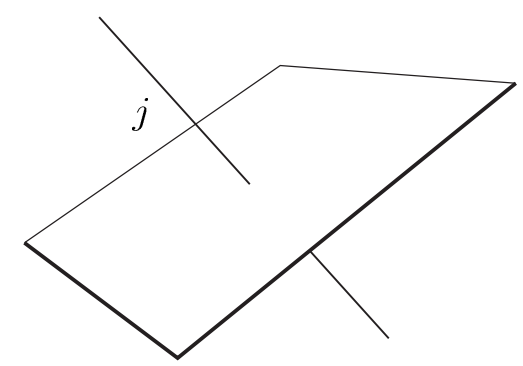

FIG. 1: In canonical quantum gravity the area of a surface is quantized.If a surface intersects a spin network edge with label $j$ it acquires an area of $8 \pi \gamma l_{P}^{2} \sqrt{j(j+1)}$. The parameter $\gamma$ is called the Immirzi parameter.

contributes the amount of area given by formula (11) to the whole area. On the horizon such a puncture also increases the dimensionality of the Hilbert space of the theory living on the boundary. Each puncture of an edge with spin $j$ increases the dimension by a factor of $2 j+1$, i.e. by the dimension of the spin $j$ representation. If there there is a large number $N$ of edges with spins $j_{i}$, $i=1, \ldots, N$, intersecting the horizon the dimension of the boundary Hilbert space is

$$
\prod_{i=1}^{N}\left(2 j_{i}+1\right)
$$

The entropy of a black hole with a given area $A$ is then given by the logarithm of the dimension of the Hilbert space of the boundary theory. It can be shown that the statistically most important contribution comes from those configurations in which the lowest possible spin dominates. Let us denote this spin by $j_{\text {min }}$. The entropy is then

$$
S=N \ln \left(2 j_{\min }+1\right)
$$

where $N$ can be calculated from the area $A$ of the black hole and from the amount of area $A\left(j_{\text {min }}\right)$ contributed by every puncture. One obtains

$$
N=\frac{A}{8 \pi l_{P}^{2} \gamma \sqrt{j_{\min }\left(j_{\min }+1\right)}}
$$




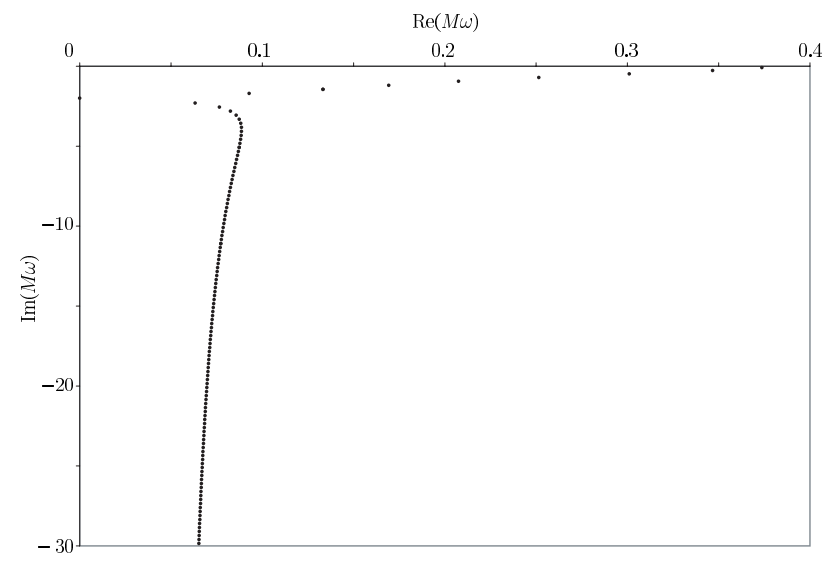

FIG. 2: This figure shows the first 124 quasinormal mode frequencies of a Schwarzschild black hole.

Equating the result of equation (3) with the known Bekenstein - Hawking result then gives a value for the Immirzi parameter. The lowest non-trivial representation of $\mathrm{SU}(2)$ has spin $j_{\min }=1 / 2$ and one obtains the value $\ln 2 / \pi \sqrt{3}$ for the Immirzi parameter.

In this note we want to fix the Immirzi parameter in a way that is independent from the black hole considerations outlined above. For this we will make use of an observation by Hod [14] on the quasinormal ringing modes of a black hole. Aside from arriving at a new value for the Immirzi parameter we will argue that the lowest admissible spin should be $j_{\min }=1$ and not $j_{\min }=1 / 2$.

\section{B. Quasinormal Modes}

The reaction of a back hole to a perturbation will be dominated by a set of damped oscillations called quasinormal modes. They appear as solutions to the perturbation equation of the Schwarzschild geometry found by Regge and Wheeler, and Zerilli. Figure 2 shows the first quasinormal mode frequencies in the complex $\omega$ plane. (See the review articles by Nollert [10], and Kokkotas and Schmidt [11] for more information about quasinormal modes.)

For large damping, i.e. for a large imaginary part of $\omega$, the real part of the frequency approaches a nonzero value and the imaginary part becomes equally spaced. Nollert [12] found the following limiting behavior of the quasinormal mode frequencies:

$$
M \omega=0.04371235+\frac{i}{4}\left(n+\frac{1}{2}\right)
$$

where $M$ is the mass of the black hole. This relation was later confirmed by Andersson [13. Crucial for our argument is an observation made by Hod 14. He remarked that the constant real part of the quasinormal frequencies is equal to

$$
\frac{\ln 3}{8 \pi}
$$

So far the evidence for this relation is purely numerical. What is remarkable here is the appearance of the $\ln 3$ term. Since we will use this frequency later on, we denote it by $\omega_{\mathrm{QNM}}$ :

$$
\omega_{\mathrm{QNM}}=\frac{\ln 3}{8 \pi M}
$$

\section{The Area Spectrum}

Using $\omega_{\mathrm{QNM}}$ we will now fix the ambiguity of the area spectrum. According to Bohr's correspondence principle an oscillatory frequency of a classical system should be equal to a transition frequency of the corresponding quantum system. The most natural candidate for a transition of the quantum black hole as described above is the appearance or disappearance of a puncture with spin $j_{\text {min }}$. The area of the black hole would then change by an amount given by equation (1) where $j=j_{\min }$ :

$$
\Delta A=A\left(j_{\min }\right)=8 \pi l_{P}^{2} \gamma \sqrt{j_{\min }\left(j_{\min }+1\right)}
$$

We can now fix the Immirzi parameter $\gamma$ appearing in this equation by requiring that the change $\Delta M$ in the mass corresponding to this change in area equals the energy of a quantum with frequency $\omega_{\mathrm{QNM}}$, i.e. we will fix $\gamma$ by setting

$$
\Delta M=\hbar \omega_{\mathrm{QNM}}=\frac{\hbar \ln 3}{8 \pi M} .
$$

Since the area $A$ and the mass $M$ of a Schwarzschild black hole are related by

$$
A=16 \pi M^{2}
$$

the mass change of equation (9) translates into the area change

$$
\Delta A=4 \ln 3 l_{P}^{2} .
$$

Comparing this with equation (8) gives the desired expression for the Immirzi parameter $\gamma$ :

$$
\gamma=\frac{\ln 3}{2 \pi \sqrt{j_{\min }\left(j_{\min }+1\right)}}
$$

In the next section we will go further and also fix $j_{\min }$.

\section{The Entropy}

The entropy is given by the logarithm of the dimension of the Hilbert space of the boundary theory. As before we have

$$
S=N \ln \left(2 j_{\min }+1\right)
$$


where $N$ is the number of punctures given by

$$
N=\frac{A}{A\left(j_{\min }\right)} .
$$

In the previous section we argued that $\Delta A=A\left(j_{\min }\right)=$ $4 \ln 3 l_{P}^{2}$ and thus

$$
S=\frac{A}{4 l_{P}^{2}} \frac{\ln \left(2 j_{\min }+1\right)}{\ln 3} .
$$

A complete agreement with the Bekenstein - Hawking result is obtained if we set

$$
j_{\text {min }}=1 .
$$

This then also fixes the value for the Immirzi parameter:

$$
\gamma=\frac{\ln 3}{2 \pi \sqrt{2}}
$$

\section{E. Discussion}

The quasinormal mode spectrum of a black hole singles out the frequency $\omega_{\mathrm{QNM}}$ of equation (7). For large damping $\omega_{\mathrm{QNM}}$ is the real part of a quasinormal mode frequency. This uniqueness of $\omega_{\mathrm{QNM}}$ strongly suggests that this frequency should also play a role in the quantum theory of a black hole. Invoking Bohr's correspondence principle we argued that $\omega_{\mathrm{QNM}}$ should appear as a transition frequency in the quantum theory.

In canonical quantum gravity the area of a macroscopically large black hole arises through a large number of intersection of the black horizon surface with spin network edges with the same low spin $j_{\min }$. The natural candidate for a quantum transition is thus the appearance or disappearance of a puncture of spin $j_{\min }$. By equating the mass change corresponding to this area change with the energy $\hbar \omega_{\mathrm{QNM}}$ of a quantum with frequency $\omega_{\mathrm{QNM}}$ we can fix the Immirzi parameter as a function of the lowest spin $j_{\min }$.

Using these results we were then able to calculate the entropy of a black hole. This entropy agrees with the Bekenstein - Hawking result provided one chooses

$$
j_{\text {min }}=1 .
$$

We want to point out how remarkable this result is. The purely classical frequency $\omega_{\mathrm{QNM}}$ contains exactly the factor of $\ln 3$ required to cancel the same factor appearing in the formula for the entropy. The classical theory and the quantum theory of a black hole conspire here to give both the Bekenstein - Hawking entropy and a prediction about the lowest admissible spin $j_{\min }$.

Next we want to discuss the significance of the result $j_{\min }=1$. There are two possible explanations. The first explanation can be that the gauge group to consider is $\mathrm{SO}(3)$ and not $\mathrm{SU}(2)$. Since the unitary representations of $\mathrm{SO}(3)$ are labelled by integers the lowest allowed spin would be $j_{\min }=1$. This explanation is perfectly plausible since the use of $\mathrm{SU}(2)$ connections is not motivated by physical consideration but rather by convenience. In most of the calculations the gauge group appears only through its Lie algebra and it is then not surprising that the representations of this algebra are used. In the case of canonical quantum gravity this means that it is not $\mathrm{SO}(3)$ that is used but its simply connected covering group $\mathrm{SU}(2)$.

Another explanation can be that there is a physical reason why spin $1 / 2$ punctures should not be counted. No such reason is known so far.

We pointed out above that the area of a macroscopically large black hole is mainly due to a large number of punctures with one low spin $j_{\min }$. This means that the possible transitions are dominated by the appearance and disappearance of one puncture with spin $j_{\min }$. We had to invoke this statistical argument here since the area spectrum obtained from (11) is nearly continuous for a large area. This is because the possible values for the area coming from one puncture depend on the spin in an irrational manner through a square root. The possible values for the area obtained by a large number of punctures then become dense for large areas.

One can argue that this means that the quantization of the area operator has to be changed. Recently such quantizations have been proposed [15]. The spectrum obtained is of the form

$$
A=\tilde{\gamma} l_{P}^{2}(j+1 / 2) .
$$

Since this spectrum is equally spaced the possible values for the area of a macroscopically large black hole are also equally spaced with the same spacing. Using the same arguments as above one can then fix the constant $\tilde{\gamma}$ to be equal to $4 \ln 3$.

The problem with this approach is that it does not give the Bekenstein - Hawking entropy if one follows the same procedure as above. Since the difference $\Delta A$ between two adjacent area values does not equal the area $A\left(j_{\min }\right)$ the entropy is not equal to $A / 4 l_{P}^{2}$. With $j_{\text {min }}$ equal to unity one obtains an entropy of $A / 6 l_{P}^{2}$. This problem arises because of the $1 / 2$ in formula (18). If one would remove this constant one would again have $\Delta A=A\left(j_{\min }\right)$ and one would again obtain the Bekenstein - Hawking result.

For the argument in this paper to work we need

$$
\Delta A=A\left(j_{\min }\right),
$$

where $\Delta A$ is equal to $4 \ln 3 l_{P}^{2}$. This was obtained from the quasinormal mode considerations.

The treatment of black holes in canonical quantum gravity is so far confined to non-rotating black holes. The inclusion of rotation has proven to be a non-trivial task. Maybe the insight gained here by looking at quasinormal modes can shed some light on the problem. The quasinormal mode spectrum of a rotating black hole is considerably more complicated than that of a non-rotating 
black hole (see the reviews 10, 11] and also the work by Leaver 17). If a connection between classical and quantum theory also holds true for rotating black holes, a more complicated set of quantum transitions is required.

When the lowest allowed spin is $1 / 2$ there is a nice similarity between the picture coming from canonical quantum gravity and Wheeler's "it from bit" picture [16]. It seems that we are led to a somewhat more complicated picture in which the degrees of freedom are not just \pm 1 as in Wheeler but $0, \pm 1$.

Finally we want to point out that the result we use in all our derivations, namely that $\omega_{\mathrm{QNM}}=\ln 3 / 8 \pi M$, is so far known only through numerical calculations. The argument would be even stronger with an analytic proof of this result.

The author would like to thank G. Kunstatter, E. R. Livine, D. Oriti, and the members of the Perimeter Institute for fruitful discussions.
[1] T. Thiemann, gr-qc/0110034.

[2] C. Rovelli and L. Smolin, Nucl. Phys. B442, 593 (1995), gr-qc/9411005.

[3] A. Ashtekar and J. Lewandowski, Class. Quantum Grav. 14, 55 (1997), gr-qc/9602046.

[4] G. Immirzi. Nucl. Phys. Proc. Suppl. 57, 65 (1997), gr-qc/9701052.

[5] L. Smolin, J. Math. Phys. 36, 6417 (1995), gr-qc/9505028.

[6] C. Rovelli, Phys. Rev. Lett. 77, 3288 (1996), gr-qc/9603063.

[7] K. Krasnov, Phys. Rev. D. 55, 3505 (1997), gr-qc/9603025.

[8] A. Ashtekar, J. Baez, A. Corichi, and K. Krasnov, Phys. Rev. Lett. 80, 904 (1998), gr-qc/9710007.
[9] A. Ashtekar, J. Baez, and K. Krasnov, Adv. Theor. Math. Phys. 4, 1 (2000), gr-qc/0005126.

[10] H.-P. Nollert, Class. Quantum Grav. 16, R159 (1999).

[11] K. D. Kokkotas and B. G. Schmidt, Living Reviews in Relativity, (1999), gr-qc/9909058.

[12] H.-P. Nollert, Phys. Rev. D 47, 5253 (1993).

[13] N. Andersson, Class. Quantum Grav. 10, L61 (1993).

[14] S. Hod, Phys. Rev. Lett. 81, 4293 (1998), gr-qc/9812002.

[15] A. Alekseev, A. P. Polychronakos, and M. Smedbäck, arXiv:hep-th/0004036

[16] J. Wheeler, in Sakharov Memorial Lectures in Physics, edited by L. Keldysh and V. Feiberg (Nova Science, New York, 1992), Vol. 2.

[17] E. W. Leaver, Proc. R. Soc. London A402, 285 (1985) 